\title{
Ultrasound-guided continuous fascia iliaca compartment block for pre-operative pain control in very elderly patients with hip fracture: A randomized controlled trial
}

\author{
YANHUI MA, JIE WU, JIXIU XUE, FEI LAN and TIANLONG WANG \\ Department of Anesthesiology, Xuanwu Hospital, Capital Medical University, Beijing 100053, P.R. China
}

Received November 8, 2017; Accepted June 1, 2018

DOI: $10.3892 / \mathrm{etm} .2018 .6417$

\begin{abstract}
The present study presented the results of a prospective, randomized controlled trial. The present study enrolled 98 very elderly patients with hip fractures, complicated with at least one cardiovascular, neurological or pulmonary disease, of whom 10 patients were excluded. A total of 88 patients were randomly assigned into 2 groups: i) The control group, receiving traditional analgesia including $50 \mathrm{mg}$ Tramadol and $500 \mathrm{mg}$ paracetamol orally three times a day from admission to surgery; and ii) the study group, receiving ultrasound-guided continuous fascia iliaca compartment block (CFICB), a single $50 \mathrm{ml} 0.4 \%$ ropivacaine injection with continuous infusion of $0.2 \%$ ropivacaine at a dose of $5 \mathrm{ml} / \mathrm{h}$ from admission to surgery. The primary outcome measure of pain relief or pain intensity was assessed preoperatively and up to $48 \mathrm{~h}$ postoperatively using a visual analog scale (VAS). The results of the present study indicated that in the preoperative period, in the morning of the day of surgery, the VAS pain at rest scores were lower in the study group compared with the control group $(\mathrm{P}=0.023)$. The VAS passive movement scores of the study group were also significantly lower compared with the control group $1 \mathrm{~h}$ following analgesia at the time of admission $(\mathrm{P}<0.05)$ and in the morning of the day of surgery $(\mathrm{P}<0.05)$. Scores for patients' satisfaction with the analgesic regimen in the preoperative period were greater in the study group compared with the control group $(\mathrm{P}<0.001)$. There was no difference in analgesia-associated side effects between groups. Duration of hospital stay of patients in the control group was significantly longer compared with the study group $(\mathrm{P}=0.001)$. Patients in the study group were less likely to have increased complications compared with patients in the control group over the N2-N4 period (from preoperative period to after surgery; $\mathrm{P}=0.016$ ). The present study concluded that ultrasound guided
\end{abstract}

Correspondence to: Professor Tianlong Wang, Department of Anesthesiology, Xuanwu Hospital, Capital Medical University, 45 Changchun Street, Xicheng, Beijing 100053, P.R. China

E-mail: wtl_dr111@163.com

Key words: hip fracture, geriatrics, pain, ultrasound-guided fascia iliaca compartment block, analgesia
CFICB was an effective method of providing analgesia for very elderly ( $\geq 80$ years old) with hip fracture.

\section{Introduction}

The number of elderly patients undergoing surgery due to hip fracture is increasing both in China and around the world (1). It is predicted that by $2050,6.3$ million new cases of hip fracture will be diagnosed each year (2). Hip fracture is common in the elderly, and is associated with significant morbidity and mortality (3). The mortality during hospital stay is $2.3-13.9 \%$, 6-month mortality ranges from $12-23 \%$, and 1-year mortality is $\sim 37.1 \%$ for men and $\sim 26.4 \%$ for women (4). The main reported causes of mortality following hip fracture are cardiovascular, neurological and pulmonary (5). Numerous guidelines and researches recommend that patients with hip fracture should undergo surgery within 24-48 h and early surgical treatment can relieve pain, and reduce the incidence of postoperative complications and mortality (6-9). Despite remarkable benefits of early surgery following hip fracture on outcomes and elderly patients exhibit numerous complications. The most common coexisting diseases include cardiovascular disease (35\%), respiratory disease (14\%) and cerebrovascular disease (13\%), and $\sim 70 \%$ of patients are classified as American Society of Anesthesiologists (ASA) stage III or IV (10) in poor health and time to surgery is prolonged due to direct oral anticoagulants and other factors, including waiting for routine medical clearance and unavailability of the operating suite or surgeon $(11,12)$. In China, very elderly are frequently defined as $\geq 80$ years old patients, and it has been estimated that the lifetime risk for an 80 -year-old man or woman to sustain a hip fracture is 9 and $19 \%$, respectively (13). A survey has indicated that very elderly patients with hip fracture in Xuanwu Hospital, Capital Medical University (Beijing, China) account for $35 \%$ of all very elderly patients, which is significantly increased compared with the numbers reported in Sweden (the percentage of very elderly patients with hip fracture in Sweden accounts for $9 \%$ for men and $19 \%$ for women of very elderly patients) (13). High morbidity and mortality are expected due to the advanced age and comorbidities of these patients (14). Preoperative management serves an important role in decreasing the morbidity and mortality of patients. Multiple interventions have been employed to minimize complications and duration of hospitalization including preoperative analgesia $(9,15)$. Severe pain is frequently observed in 
the preoperative period. Preoperative analgesia is administered to relieve pain and decrease the systemic stress response (16). Systemic analgesia using opioids and nonsteroidal analgesia may lead to significant adverse effects particularly in elderly patients due to age-associated pharmacokinetic and pharmacodynamic alterations (17). Previous studies have demonstrated the advantages of epidural analgesia by comparing epidural analgesia with conventional drug analgesia in elderly patients with hip fractures $(18,19)$. The results of these studies indicated that epidural analgesia can reduce the incidence of preoperative cardiovascular events, however, epidural block has an impact on circulation, and patients with hip fracture receive anticoagulant therapy immediately following admission, which may lead to epidural hematoma and other severe consequences $(18,19)$. Peripheral nerve blockade is a potential substitute for epidural analgesia (20), which requires further study. Due to the advantages of fascia iliaca compartment block, its application in analgesia prior to hip fracture surgery is increasing, but a number of studies have investigated the effect of single fascia iliaca compartment block $(21,22)$. Very elderly patients mostly exhibit one or more comorbidities and, therefore, the preoperative waiting time may be prolonged. In these cases continuous fascia iliaca compartment block (CFICB) may be more advantageous and two studies have indicated that CFICB is effective in hip fracture analgesia $(23,24)$. However, whether ultrasound-guided CFICB is more advantageous compared with traditional analgesic drugs and whether it helps to reduce the incidence of perioperative complications in very elderly patients complicated with cardiovascular, neurological or pulmonary disease have not yet been reported. It is hypothesized that fascia iliaca blocks by means of continuous catheters may greatly reduce the morbidity in patients with hip fracture for the evaluation of the influence and prevalence of resultant side effects from opiate medications. It is therefore of interest to evaluate the effectiveness of preoperative analgesia provided by CFICB or systemic analgesia in very elderly patients with hip fracture. The aim of the present study was to investigate the effect of ultrasound-guided CFICB in very elderly patients with hip fracture complicated with cardiovascular, neurological or pulmonary disease in the preoperative waiting period.

\section{Patients and methods}

Patients. The present study was prospective, randomized and controlled, and had been registered at www.chictr.org.cn (registration number: ChiCTR-IPR-15007283). This study was approved by the Ethics Committee of Xuanwu Hospital of Capital Medical University and all patients had signed informed consent. Elderly patients admitted to Xuanwu Hospital, Capital Medical University (Beijing, China) due to hip fracture from October 2015 to December 2016 were included in the present study (Fig. 1). The following inclusion criteria were used in the present study: i) Diagnosis of hip fracture (femoral neck or intertrochanteric fractures) by X-ray at admission; ii) age $\geq 80$ years; iii) complication with at least one kind of cardiovascular, neurological or pulmonary disease; iv) ASA classification III or IV; and iv) being male or female. Exclusion criteria were: i) Presence of more than one fracture; ii) allergy to amides, paracetamol and tramadol; iii) infection at fascia iliaca compartment puncture site; iv) peripheral neuropathy; v) contraindication of intraspinal block; vi) renal insufficiency and dementia; vii) preoperative waiting time $\geq 5$ days; and vii) refusing to participate in this study.

Treatment groups and the protocol. Patients meeting the inclusion criteria were randomly assigned into two groups, the control group and the study group, by a computer-generated random number table method (MATLAB 2014a; MathWorks, Natick, MA, USA) with randomized group information sealed in an opaque envelope, the envelopes were numbered and used sequentially. All patients were assessed cognitive function with the Mini-Mental State Examination at admission. The control group received traditional analgesia including $50 \mathrm{mg}$ Tramadol (batch no. 10087215; Mundipharma International, Ltd., Cambridge, UK) and $500 \mathrm{mg}$ paracetamol (batch no. 140616620; Johnson \& Johnson, New Brunswick, NJ, USA) orally three times a day from admission to surgery. The patients in the control group were not subjected to CFICB and were administrated with saline. In the study group, after the patients were diagnosed with hip fracture by X-ray at admission, the fracture side received ultrasound-guided CFICB following establishment of the upper limb venous pathway. The CFICB operation was performed as follows: Patients were in supine position and the puncture site was selected at $1 \mathrm{~cm}$ to the junction point at $1 / 3$ of the connection of anterior superior spine and pubic tubercle. A M-Turbo ultrasound apparatus (Sonosite, Inc., Bothell, WA, USA) high-frequency probe was placed in parallel to the inguinal fold to distinguish the femoral fascia and fascia iliaca, the needle was inserted with out-of-plane technique at an angle of $45^{\circ}$ and the tip of the needle was pointed to the head. Once needle-tip placement under the fascia iliaca by hydrolocation was confirmed, the probe was rotated $90^{\circ}$ into a longitudinal parasagittal orientation to visualize the needle tip in-plane and to track cephalad spread of the injectate under fascia iliaca. A total of $50 \mathrm{ml} 0.4 \%$ ropivacaine (batch no. NAKR; AstraZeneca, Cambridge, UK) was injected and formed a fluid-filled space beneath the fascia. Subsequently, the catheter was inserted with the puncture needle and the tip of the catheter was $5-10 \mathrm{~cm}$ beyond the tip of the needle. Following confirmation of the correct location of the catheter, the catheter was fixed tightly and connected to the electronic analgesic pump to continuously infuse $0.2 \%$ ropivacaine at a rate of $5 \mathrm{ml} / \mathrm{h}$ until the day of surgery. The local anesthetic moved cephalad and reached the nerves of the lumbar plexus, including the femoral, lateral cutaneous and obturator. If the analgesic effect was inadequate (VAS score $>30 \mathrm{~mm}$ ), $5 \mathrm{ml}$ of $0.2 \%$ ropivacaine would be added into the fascia iliaca compartment. In the morning of the day of surgery, the catheter was removed from the fascia iliaca compartment. Ultrasound-guided CFICB was performed by an anesthesiologist with experience in ultrasound-guided nerve block.

According to the type of fracture, the patients were treated with proximal femoral nail antirotation, hemiarthroplasty, cannulated screws or total hip replacement. Subcutaneous injection of enoxaparin sodium (40 mg/day; batch no. 4sj11; Sanofi S.A., Paris, France) was given to all patients preventively after admission, which was discontinued $12 \mathrm{~h}$ prior to surgery and continued on the first day after surgery. All patients received combined subarachnoid and epidural anesthesia in L2-3 or L3-4 interspace and subarachnoid injection 


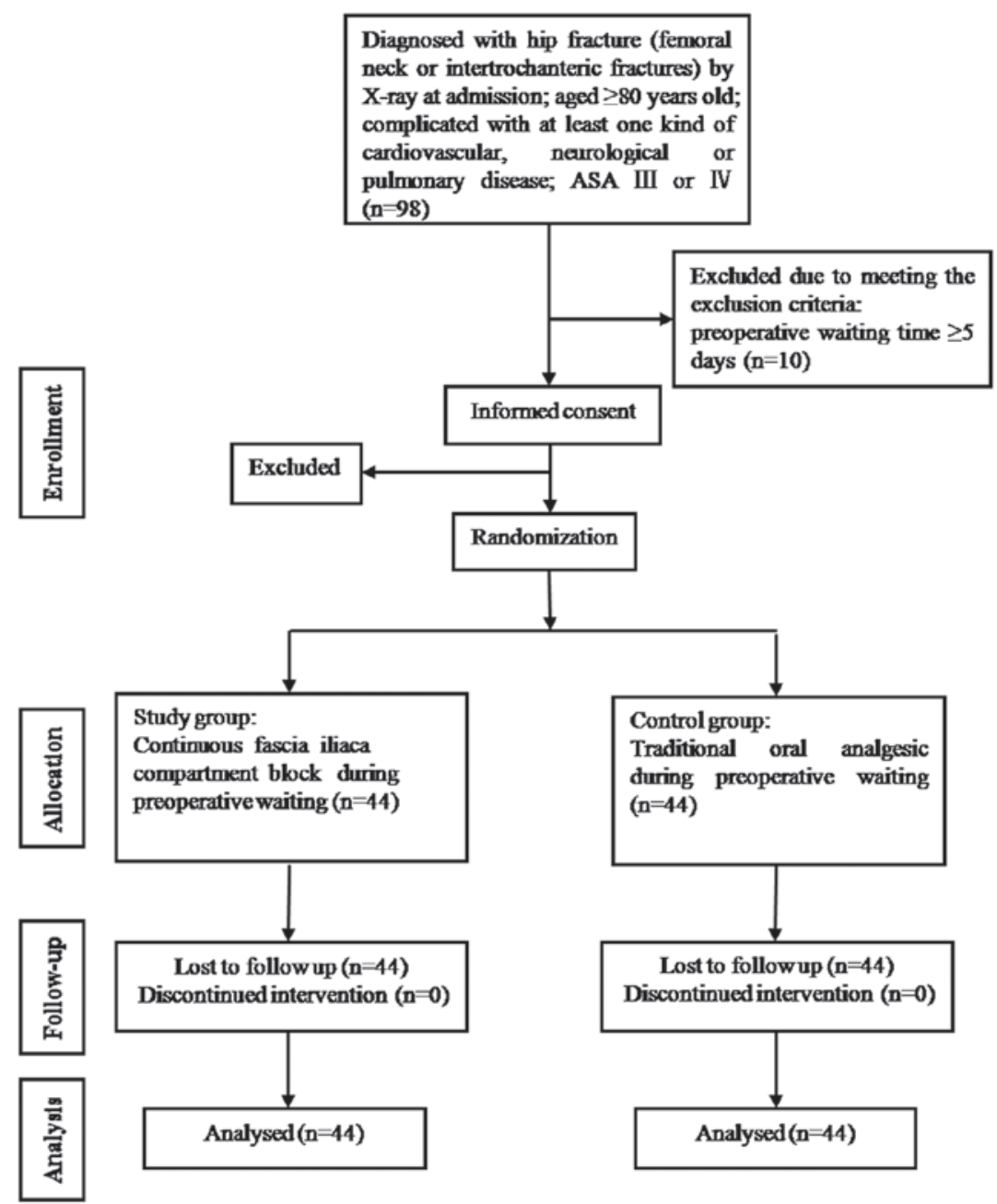

Figure 1. Consolidated standards of reporting trials flowchart of subject enrollment.

of $0.5 \%$ bupivacaine (batch no. 73141014; Shanghai Harvest Pharmaceutical Co., Ltd, Shanghai, China) 1.0-1.5 ml for $35-40 \mathrm{sec}$. After the injection, a $3 \mathrm{~cm}$ catheter was placed into the epidural cavity, 2\% lidocaine (batch no. 20150401; Beijing Yimin Pharmaceutical Co., Ltd, Beijing, China) was administered through the epidural catheter in doses of $3 \mathrm{ml}$ as needed, and the level of anesthesia was maintained at $\sim \mathrm{T}_{10}$. After surgery, all patients entered the post anesthesia care unit for at least $1 \mathrm{~h}$ and then were transferred to the wards. All patients received patient-controlled intravenous analgesia within $48 \mathrm{~h}$ after surgery, and the analgesic regimen was $1.5 \mu \mathrm{g} / \mathrm{kg}$ sufentanil (batch no. 1150309; Yichang Humanwell Pharmaceutical Co., Ltd, Yichang, China) and $8 \mathrm{mg}$ ondansetron hydrochloride (batch no. 140202A02; Ningbo Tenet Pharmaceutical Co., Ltd, Zhenhai, China), diluted with normal saline to $100 \mathrm{ml}$, at a background dose of $1-2 \mathrm{ml} / \mathrm{h}$ and a single added dose of $1 \mathrm{ml}$, with a lockout interval of $10 \mathrm{~min}$. From day 3 to discharge, the patients received oral analgesic drugs.

Primary outcome measure. The present study used VAS scores (0-100, with 0 indicating no pain and 100 indicating the worst imaginable pain) to evaluate the analgesic effect and recorded VAS scores at rest (lying flat) and on movement (movement-associated pain was elicited by passively elevating the fractured leg to $15^{\circ}$ ) prior to administration of analgesia ( $\left.\mathrm{t} 0\right), 1 \mathrm{~h}$ after administration of analgesia (t1), in the morning of the day of surgery (before surgery; $\mathrm{t} 2$ ), in the morning of the day after surgery (t3) and in the second morning after the day of surgery (t4). The patients used VAS (0-100) to evaluate the satisfaction with the analgesic regimen in the preoperative waiting period.

Secondary outcome measures. The present study recorded the number of patients developing analgesia-induced complications, including nausea, vomiting, respiratory depression (respiratory rate of $<10 / \mathrm{min}$ ) and excessive sedation (Ramsay score $\geq 3$ points, where score 1 point indicated not quiet, agitated patients; 2 points: Quiet and cooperative patients; 3 points: Drowsy and obeying directive; 4 points: Drowsy and responding to tactile stimulation; 5 points: Lethargic and only responding to strong stimulation; 6 points: In deep sleep, no response to calling). The number of patients needing additional rescue analgesia and CFICB complications were also recorded, including local anesthetic toxicity, puncture site infection and hematoma. Nausea and/or vomiting were treated using intravenous administration of ondansetron. Respiratory depression was treated by close surveillance together with naloxone and oxygen.

Other outcome measures. Incidence of cardiovascular, pulmonary and cerebral complications during hospital stay was 
also recorded. Cardiovascular complications included acute myocardial infarction, angina pectoris, new arrhythmia (atrial fibrillation, ventricular premature beats and tachycardia), acute heart failure, cardiac arrest and deep venous thrombosis of lower extremities. Diagnosis of myocardial infarction was reached if: i) CK-MB isoenzyme or troponin-I concentrations were above the laboratory myocardial infarction threshold (CK-MB, $>4.99 \mathrm{ng} / \mathrm{ml}$; cTNI, $>0.02 \mathrm{ng} / \mathrm{ml}$ ); and ii) either new $\mathrm{Q}$ waves ( $\geq 0.03 \mathrm{~s}$ ) or persistent alterations (4 days) were identified in the ST-T segment. Unstable angina was defined as severe precordial chest pain with a duration of $\geq 30 \mathrm{~min}$ and unresponsive to standard therapy, associated with ST-segment or T-wave alterations without Q waves or cardiac enzyme abnormalities. Congestive heart failure was defined using clinical (shortness of breath, rales, jugular venous distention, peripheral edema and third heart sound) and radiological (cardiomegaly, interstitial edema, alveolar edema) signs and required modified medication involving minimum treatment with diuretic drugs. New arrhythmia was confirmed by 12-lead electrocardiogram. Pulmonary complications included pulmonary infection, pulmonary embolism and respiratory failure, and cerebral complications included new cerebral infarction and delirium. The above complications were diagnosed by clinical symptoms associated with relevant auxiliary examinations. The incidence of cardiovascular, pulmonary and cerebral complications was recorded in three periods: The preoperative waiting period, surgery and form the end of surgery to discharge. Four different periods, including hospital admission, preoperative period, surgery and following surgery, were denoted as N1, N2, N3 and N4, respectively. In addition, the preoperative waiting time, length of stay, hospital costs and mortality during hospitalization were recorded.

Analgesic effects, analgesia-induced complications and CFICB-associated complications were evaluated and recorded by the acute pain service. Patients' general characteristics, including gender, age, weight, height, ASA grade and type of surgery; perioperative complications; preoperative waiting time; length of stay; hospital costs and other data were collected by another anesthesiologist in a blinded manner.

Statistical analysis. All the analyses were performed using SPSS software (version 18.0; SPSS, Inc., Chicago, IL, USA). The sample size was estimated based on two-way repeated measures analysis of variance comparison of the VAS scores between groups. VAS scores were measured at five different time points outlined above for each patient. A total of 40 patients in each group were required to complete the study and the assumptions of 5\% type I error rate, $80 \%$ power and 0.45 effect size were considered. Based on the assumption of a dropout rate of $\sim 10 \%, 44$ patients would be required to be enrolled in each group. A total of 88 patients were included in the statistical analysis, 44 cases were included in the control group and 44 cases in the study group.

Data are presented as mean \pm standard deviation for continuous variables and as frequency (\%) for categorical variables. For continuous variables, the normal distribution assumption was assessed. Equal variance assumption was also assessed. The differences of these characteristics between the control group and study group were performed using independent two-sample t-tests. Categorical variables were summarized using frequency and percentage and compared using Chi-square test. Intergroup differences in VAS scores were assessed by two-way repeated measures analysis of variance with Bonferroni post hoc test. $\mathrm{P}<0.05$ was considered to indicate a statistically significant difference.

\section{Results}

Demographic data. From October 2015 to December 2016, a total of 88 patients were included in the statistical analysis, 44 cases were included in the control group and 44 cases in the study group. General patients' characteristic and surgical characteristics were summarized in Table I. No significant differences were identified between the two groups, including age, height, weight and ASA classification (Table I). The duration of hospital stay of the control group was significantly longer compared with the study group.

Pain assessment. Although the differences were small, VAS pain scores at rest of the study group were lower compared with the control group $(\mathrm{P}=0.023)$ at the time point $\mathrm{t} 2$. There were no significant differences in the VAS pain scores at rest between the groups at the other time points $(\mathrm{P}=0.178$ at $\mathrm{t} 0$; $\mathrm{P}=0.260$ at $\mathrm{t} 1 ; \mathrm{P}=0.240$ at $\mathrm{t} 3 ; \mathrm{P}=0.103$ at $\mathrm{t} 4$; Fig. 2; Data are presented as the mean \pm standard deviation).

VAS pain scores at passive movement of the study group were significantly lower compared with the control group at the time point $\mathrm{t} 1(\mathrm{P}<0.05)$ and $\mathrm{t} 2(\mathrm{P}<0.05)$. There were no significant differences in VAS pain scores at passive movement between the groups at the other time points $(\mathrm{P}=0.931$ at $\mathrm{t} 0 ; \mathrm{P}=0.762$ at $\mathrm{t} 3$; and $\mathrm{P}=0.139$ at $\mathrm{t} 4$; Fig. 3 ; Data are presented as mean \pm standard deviation).

Scores for patients' satisfaction with the analgesic regimen in the preoperative period were increased in the study group compared with the control group $(45.68 \pm 11.29$ in the control group vs. $74.77 \pm 9.52$ in the study group; $\mathrm{P}<0.001$; Table II).

Intergroup differences in VAS scores were assessed by two-way repeated measures analysis of variance with Bonferroni post hoc test. For VAS pain scores at rest, there were no significant differences between the groups $(\mathrm{P}=0.472)$. Significant time effect $(\mathrm{P}<0.001)$ and significant interaction effect between time and group $(\mathrm{P}=0.034)$ were identified; a significant difference of VAS at rest between groups at time point $\mathrm{t} 2$ and no significant difference at other time points were observed. For VAS pain scores at passive movement, there were significant differences between groups $(\mathrm{P}<0.001)$, significant time effect $(\mathrm{P}<0.001)$ and significant interaction effect between time and group $(\mathrm{P}<0.001)$; a significant difference of VAS at passive movement between groups at $t 1$ and t2 and no significant difference at other time points were suggested.

Side effects. The incidence of nausea and vomiting were similar between the control group and the study group in the preoperative period $(\mathrm{P}=0.195$ and $\mathrm{P}=1$; respectively; Table II). All patients in the present study did not demonstrate symptoms of respiratory depression and excessive sedation in the preoperative waiting period. The study group did not develop CFICB complications (local anesthetic toxicity, puncture site infection and hematoma in preoperative waiting period; data not shown). 
Table I. Characteristics of patients.

\begin{tabular}{|c|c|c|c|}
\hline Characteristics & Control group $(n=44)$ & Study group $(n=44)$ & P-value \\
\hline Waiting time before surgery (days) & $3.34 \pm 0.89$ & $3.27 \pm 0.82$ & 0.709 \\
\hline Hospital stay (days) & $13.55 \pm 6.03$ & $9.98 \pm 2.46$ & 0.001 \\
\hline Hospital expenses (ҰRMB) & $65,786.82 \pm 19,741.77$ & $61,425.02 \pm 13,159.95$ & 0.226 \\
\hline Male $(\mathrm{n}, \%)$ & $14(31.8 \%)$ & $16(36.4 \%)$ & 0.653 \\
\hline Age (years) & $83.73 \pm 3.40$ & $84.05 \pm 5.04$ & 0.730 \\
\hline Weight (kg) & $60.18 \pm 10.83$ & $58.70 \pm 12.35$ & 0.552 \\
\hline Height (cm) & $159.93 \pm 7.12$ & $163.20 \pm 9.38$ & 0.069 \\
\hline ASA stage (n) & & & 0.676 \\
\hline III & 40 & 42 & \\
\hline IV & 4 & 2 & \\
\hline Type of surgery (n) & & & 0.752 \\
\hline Hemiarthroplasty & 9 & 6 & \\
\hline Proximal femoral nail antirotation & 33 & 34 & \\
\hline Cannulated screws & 1 & 2 & \\
\hline Total hip replacement & 1 & 2 & \\
\hline Anesthesia method (n) & & & 1.000 \\
\hline Combined spinal-epidural anesthesia & 44 & 44 & \\
\hline Duration of anesthesia (min) & $168.73 \pm 47.13$ & $158.48 \pm 36.71$ & 0.258 \\
\hline Duration of operation (min) & $95.07 \pm 42.53$ & $82.25 \pm 32.79$ & 0.117 \\
\hline Total liquid intake (ml) & $985.23 \pm 156.48$ & $965.91 \pm 158.40$ & 0.566 \\
\hline Crystalloid solution volume (ml) & $628.41 \pm 238.06$ & $613.64 \pm 206.40$ & 0.757 \\
\hline Colloidal solution volume (ml) & $279.55 \pm 241.68$ & $304.55 \pm 263.21$ & 0.644 \\
\hline Bleeding $(\mathrm{ml})$ & $180.68 \pm 89.76$ & $168.18 \pm 111.13$ & 0.563 \\
\hline Urine volume (ml) & $411.59 \pm 179.29$ & $373.86 \pm 199.27$ & 0.353 \\
\hline Intraoperative blood infusion $(\mathrm{n}, \%)$ & $9(20.5 \%)$ & $9(20.5 \%)$ & 1.000 \\
\hline MMSE & $20.95 \pm 1.57$ & $21.18 \pm 1.77$ & 0.526 \\
\hline
\end{tabular}

Values are presented as the mean \pm standard deviation or number (proportion). ASA, American Society of Anesthesiologists; MMSE, mini-mental state examination.

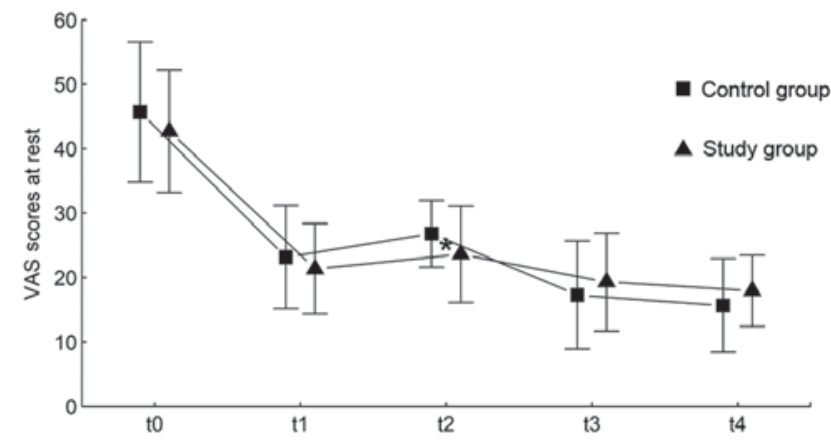

Figure 2. VAS pain scores at rest. VAS, visual analog scale; t0, VAS score prior to analgesia at basic rest; t1, VAS score $1 \mathrm{~h}$ after analgesia; $\mathrm{t} 2$, VAS score in the morning of the day of surgery; $\mathrm{t} 3$, VAS score in the first day after surgery; $\mathrm{t} 4$, VAS score in the second day after surgery. ${ }^{*} \mathrm{P}<0.05$ vs. the control group.

Number of complications. The number of complications was calculated at four different periods, including hospital admission, preoperative period, surgery and following surgery, and were denoted as N1, N2, N3 and N4, respectively (Fig. 4). The alterations in the number

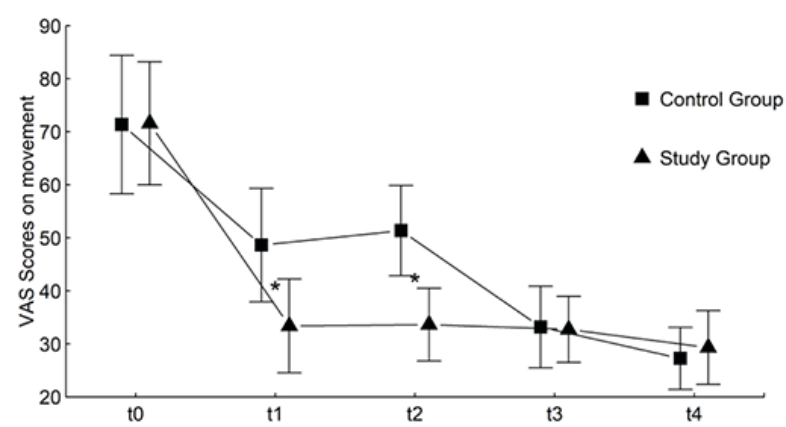

Figure 3. VAS pain scores at passive movement. t0, VAS score prior to analgesiaat basic rest; $\mathrm{t} 1$, VAS score $1 \mathrm{~h}$ after analgesia; 2 , VAS score in the morning of the day of surgery; $\mathrm{t} 3$, VAS score in the first day after surgery; $\mathrm{t} 4$, VAS score in the second day after surgery. ${ }^{*} \mathrm{P}<0.05$ vs. the control group.

of complications between two different time points were analyzed, as shown in Table III. Patients in the study group were less likely to have increased complications compared with patients in the control group over the N2-N4 period (from preoperative period to after surgery; $\mathrm{P}=0.016$; Table III). 
Table II. Analgesia satisfaction scores and analgesia-associated side effects.

\begin{tabular}{|c|c|c|c|}
\hline Parameters & Control group $(n=44)$ & Study group $(n=44)$ & P-value \\
\hline Satisfaction with the analgesic regimen in the preoperative period & $45.68 \pm 11.29$ & $74.77 \pm 9.52$ & $<0.001$ \\
\hline Nausea $(n, \%)$ & $12(27.3 \%)$ & $7(15.9 \%)$ & 0.195 \\
\hline Vomiting $(\mathrm{n}, \%)$ & $5(11.4 \%)$ & $4(9.1 \%)$ & 1.000 \\
\hline
\end{tabular}

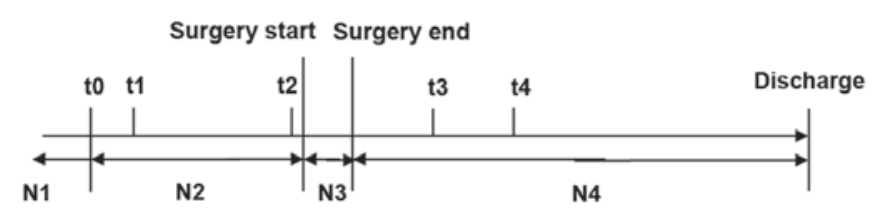

Figure 4. Analgesic effect observation time points (t0-4) and complications observation time periods (N1-4).

Patient mortality. A total of 3 patients succumbed to mortality in postoperative hospitalization. A total of 2 cases $(4.5 \%)$ succumbed to mortality in the control group, including 1 mortality due to respiratory failure and 1 mortality due to acute myocardial infarction and heart failure. A patient $(2.3 \%)$ in the study group succumbed to sudden cardiac mortality associated with malignant arrhythmia.

\section{Discussion}

Patients with hip fracture are often in considerable pain and, if untreated, may develop various complications that delay operative intervention and prolong hospitalization (25). Pain management is believed to be critical in both the pre- and post-operative periods for patients with geriatric hip fractures (26-28). For most fractures, administration of systemic analgesics including opioids, paracetamol and non-steroidal anti-inflammatory drugs is preferred for pain management. Despite the relative safety of paracetamol, both opioids and nonsteroidal analgesia can induce significant adverse effects especially in the elderly population $(17,29)$. According to the guidelines from the National Institute for Health and Clinical Excellence, neural blockade by trained personnel is recommended for a reasonable opioid dosage (30).

The present study demonstrated that pain relief was superior in the study group (patients aged $\geq 80$ years old, complicated with at least one type of cardiovascular, neurological or pulmonary disease), compared with the control group, during the preoperative period. In addition, CFICB was associated with greater patient satisfaction, which was consistent with the results of Candal-Couto et al (23) and Dulaney-Cripe et al (24). Diakomi et al (31) concluded that performing an FICB prior to positioning for spinal anesthesia provided superior pain management compared with intravenous fentanyl administration facilitated spinal performance. According to Diakomi et al (31) and the present study, FICB provided superior analgesia not only prior to positioning for spinal anesthesia but also during the preoperative period. The study group in the present study did not develop CFICB-associated complications. The present results suggested that a fascia iliaca compartment block performed via a catheter provided a significant benefit to very
Table III. Number of complications in the four time periods in the control and study groups.

\begin{tabular}{|c|c|c|c|}
\hline $\begin{array}{l}\text { Time } \\
\text { period }\end{array}$ & $\begin{array}{c}\text { Control } \\
\text { group }(n=44)\end{array}$ & $\begin{array}{c}\text { Study } \\
\text { group }(n=44)\end{array}$ & P-value \\
\hline N2 vs. N1 & & & 1.000 \\
\hline No change & 40 & 41 & \\
\hline Increase & 4 & 3 & \\
\hline N3 vs. N1 & & & 1.000 \\
\hline No change & 40 & 40 & \\
\hline Increase & 4 & 4 & \\
\hline N4 vs. N1 & & & 0.059 \\
\hline No change & 32 & 39 & \\
\hline Increase & 12 & 5 & \\
\hline N3 vs. N2 & & & 0.306 \\
\hline Decrease & 2 & 0 & \\
\hline No change & 41 & 43 & \\
\hline Increase & 1 & 1 & \\
\hline N4 vs. N2 & & & 0.016 \\
\hline Decrease & 2 & 0 & \\
\hline No change & 33 & 42 & \\
\hline Increase & 9 & 2 & \\
\hline
\end{tabular}

$\mathrm{N} 1$, hospital admission; N2, preoperative period; N3, during surgery; N4, after surgery.

elderly patients with hip fractures. Hip innervation involves the lateral cutaneous nerve of the thigh, the femoral nerve and the obturator nerve (32). Fascia iliaca compartment block utilizes the space under the iliac fascia where the femoral, obturator and lateral cutaneous nerves are located (33). The block acts by suppressing the sensation of pain in the femoral nerve, lateral femoral cutaneous nerve of the thigh and obturator nerve (22). The incidence of nausea and vomiting were similar between the control group and the study group during pre-operative period, which may have been due to the small number of patients enrolled in this study. In other countries, a majority of patients with hip fracture undergo single fascia iliaca compartment block in the emergency room prior to surgery $(21,22)$, while in the present study, continuous block and perineural catheter placement permitted the provision of continuous peripheral nerve block, thereby extending the duration of analgesia.

Szucs et al (34) have demonstrated that continuous femoral nerve block provided a more effective perioperative analgesia compared with a standard opiate-based regimen for patients undergoing fixation of fractured neck of femur. Another study 
suggested that both ultrasound-guided continuous femoral nerve block and fascia iliaca compartment block with a novel cannula-over-needle provide effective anesthesia and postoperative analgesia for elderly patients with hip replacement (35). It has been reported that 3-in-1 femoral nerve block is also effective in hip fracture analgesia $(20,36)$. Femoral nerve block is recognized as an effective method; however, it is controversial due to its potential for nerve injury and femoral nerve block can cause quadriceps weakness (37). The FICB technique is somehow favorable compared with the femoral nerve block technique, as it is not necessary to use a neurostimulator during the procedure (38). The site of injection with the FICB is distant from any nerves or blood vessels, where intravascular or intraneural injection is contraindicated in principle (39). The fascia iliaca compartment block is more effective compared with the 3 -in-1 block in producing simultaneous blockade of the lateral femoral cutaneous nerve of the thigh and femoral nerves in adults (40). The three nerves, including femoral nerve, lateral femoral cutaneous nerve of the thigh and obturator nerve, were more successfully blocked by FICB (>90\%) compared with the 3 -in-1 block (20\%) (33). In addition, performing the fascia iliaca compartment block is easier to teach and learn compared with the 3-in-1 block, and more convenient and cost-effective (41). Therefore, the continuous FICB was selected in the present study.

The sensorial innervation of the hip is provided by the nerves in the lumbar plexus and sacral plexus (42). Although whether lumbar or sacral plexus have a primary effect on sensorial innervation of the hip remains unknown, according some previous studies $(39,40)$ and the present study, the lumbar plexus block can provide effective analgesia. In addition, the two groups of patients included in the present study did not undergo FICB after surgery, as neural blockade could intervene with postoperative mobilization in those patients.

There are numerous ways to perform the FICB, including the loss of resistance ('2-pop technique') (21), ultrasound guided blocks (43) or the nerve stimulators for femoral nerve localization (44). Nerve stimulator-based femoral nerve block is characterized by stimulation of the leg muscles, which may cause discomfort to the patient and compromise the fracture alignment (45). Ultrasound guidance does not require a nerve stimulator, facilitates the FICB technique and reduces the risk of block failure or nerve injury $(43,46)$.

The results of the present study indicated that the length of stay was shorter and costs of hospitalization were slightly lower in the study group compared with the control group. After hip fracture, pain is an important determinant of function, which therefore determines length of stay and clinical outcome, defined by complication and mortality rates (47). Adequate analgesia allows for increased mobility of patients and reduced duration of hospitalization $(24,28)$. A shorter stay is beneficial to patients in terms of reduced risk of hospital-acquired complications and the associated reduction of costs of hospitalization is economically beneficial for the healthcare system $(24,48)$. In addition, in the present study, the duration of hospital stay was shorter in the study group compared with the control group, which may have been associated with the number of patients with increased complications being smaller in the study group compared with the control group.
Patients in the study group were less likely to have increased complications compared with patients in the control group over the N2-N4 period (from preoperative period to after surgery), and the results of the analysis of postoperative morbidity outcomes should be interpreted cautiously, as these factors, including the supplement of oxygen, fluid management and mobilization, were not controlled. However, failure to stabilize the medical conditions prior to surgery poses an increased risk of postoperative cardiac and pulmonary complications, prolonged hospitalization and mortality (49). The present study indicated that ultrasound-guided CFICB induced a satisfactory analgesic effect, which may be associated with decreased incidence of complications in the study group, compared with the control group after surgery.

In addition, mortality during hospital stay was 2/44 (4.5\%) in the control group and 1/44 (2.3\%) in the study group, which was consistent with earlier studies that reported in-hospital mortality rates of 2.3-13.9\% in unselected groups of patients with hip fracture (4).

There are several limitations of the present study and it was mainly limited by its size. Due to a small number of cases, whether ultrasound-guided CFICB can reduce the incidence of postoperative cardiovascular, pulmonary and cerebral complications in very elderly patients with hip fracture requires further confirmation by multicenter and large-sample size studies. Furthermore, the patients were not blind to group allocation due to the ethical considerations. As it was considered ethically unacceptable to insert a placebo fascia iliaca compartment catheter for blinding purposes only, no further damage could be simulated in the control group patients, and, therefore the patients in the control group were not subjected to CFICB and administrated with saline. Furthermore, the present study only recorded patients' mortality during the hospital stay, without a follow up for a longer period.

In conclusion, the present study demonstrated that ultrasound guided continuous fascia iliaca compartment block may provide a superior analgesia for very elderly patients with hip fracture compared with patients receiving systemic analgesia-based regimen during the preoperative period.

\section{Acknowledgements}

Not applicable.

\section{Funding}

The present study was supported by Beijing Municipal Administration of Hospitals' Ascent Plan (grant no. DFL20150802) and Beijing Municipal Commission of Health and Family Planning (grant no. PXM2017_026283_000002).

\section{Availability of data and materials}

The datasets used and/or analyzed during the present study are available from the corresponding author on reasonable request.

\section{Authors' contributions}

YM and TW designed the study. JW performed the collection and entry of the data. JX analyzed the data. YM prepared the 
manuscript. FL participated in the collection of the data and a literature analysis. All the authors read and approved the final manuscript.

\section{Ethics approval and consent to participate}

The study was approved by the Ethics Committee of Xuanwu Hospital, Capital Medical University (Beijing, China). Signed written informed consents were obtained from the patients.

\section{Patient consent for publication}

Not applicable.

\section{Competing interests}

The authors declare that they have no competing interests.

\section{References}

1. Johnell $\mathrm{O}$ and Kanis JA: An estimate of the worldwide prevalence, mortality and disability associated with hip fracture. Osteoporos Int 15: 897-902, 2004.

2. Watters CL and Moran WP: Hip fractures-a joint effort. Orthop Nurs 25: 157-165; quiz 166-167, 2006.

3. Marks R: Hip fracture epidemiological trends, outcomes, and risk factors, 1970-2009. Int J Gen Med 3: 1-17, 2010.

4. Boddaert J, Cohen-Bittan J, Khiami F, Khiami F, Le Manach Y, Raus M, Beinis JY, Verny M and Riou B: Postoperative admission to a dedicated geriatric unit decreases mortality in elderly patients with hip fracture. PLoS One 9: e83795, 2014.

5. Rosencher N, Vielpeau C, Emmerich J, Fagnani F and Samama CM; ESCORTE group: Venous thromboembolism and mortality after hip fracture surgery: The ESCORTE study. J Thromb Haemost 3: 2006-2014, 2005.

6. Mak JC, Cameron ID and March LM; National health and medical research council: Evidence-based guidelines for the management of hip fractures in older persons: An update. Med J Aust 192: 37-41, 2010.

7. Simunovic N, Devereaux PJ, Sprague S, Guyatt GH, Schemitsch E, Debeer J and Bhandari M: Effect of early surgery after hip fracture on mortality and complications: Systematic review and meta-analysis. CMAJ 182: 1609-1616, 2010.

8. Moja L, Piatti A, Pecoraro V, Ricci C, Virgili G, Salanti G, Germagnoli L, Liberati A and Banfi G: Timing matters in hip fracture surgery: Patients operated within 48 hours have better outcomes. A meta-analysis and meta-regression of over 190,000 patients. PLoS One 7: e46175, 2012.

9. Colais P, Di Martino M, Fusco D, Perucci CA and Davoli M: The effect of early surgery after hip fracture on 1-year mortality. BMC Geriatr 15: 141, 2015.

10. Association of Anaesthetists of Great Britain and Ireland, Griffiths R, Alper J, Beckingsale A, Goldhill D, Heyburn G, Holloway J, Leaper E, Parker M, Ridgway S, et al: Management of proximal femoral fractures 2011: Association of Anaesthetists of Great Britain and Ireland. Anaesthesia 67: 85-98, 2012.

11. Tran T, Delluc A, de Wit C, Petrcich W, Le Gal G and Carrier M: The impact of oral anticoagulation on time to surgery in patients hospitalized with hip0 fracture. Thromb Res 136: 962-965, 2015.

12. Orosz GM, Hannan EL, Magaziner J, Koval K, Gilbert M, Aufses A, Straus E, Vespe E and Siu AL: Hip fracture in the older patient: Reasons for delay in hospitalization and timing of surgical repair. J Am Geriatr Soc 50: 1336-1340, 2002.

13. Kanis JA, Johnell O, Oden A, Sembo L, Redlund-Johnell L, Dawson A, De Laet C and Jonsson B: Long-term risk of osteoporotic fracture in Malmö. Osteoporos Int 11: 669-674, 2000.

14. Roche JJ, Wenn RT, Sahota O and Moran CG: Effect of comorbidities and postoperative complications on mortality after hip fracture in elderly people: Prospective observational cohort study. Bmj 331: 1374, 2005.

15. Jandziol AK and Griffiths R: The anaesthetic management of patients with hip fractures. Bja Cepd Reviews 1: 52-55, 2001.
16. Sciard D, Cattano D, Hussain $M$ and Rosenstein A: Perioperative management of proximal hip fractures in the elderly: The surgeon and the anesthesiologist. Minerva Anestesiol 77: 715-722, 2011.

17. Godoy Monzón D, Vazquez J, Jauregui JR and Iserson KV: Pain treatment in post-traumatic hip fracture in the elderly: Regional block vs. Systemic non-steroidal analgesics. Int J Emerg Med 3: 321-325, 2010.

18. Scheinin H, Virtanen T, Kentala E, Uotila P, Laitio T, Hartiala J, Heikkilä H, Sariola-Heinonen K, Pullisaar O, Yli-Mäyry S and Jalonen J: Epidural infusion of bupivacaine and fentanyl reduces perioperative myocardial ischaemia in elderly patients with hip fracture-a randomized controlled trial. Acta Anaesthesiol Scand 44: 1061-1070, 2000.

19. Matot I, Oppenheim-Eden A, Ratrot R, Davidson E, Eylon S, Peyser A and Liebergall M: Preoperative cardiac events in elderly patients with hip fracture randomized to epidural or conventional analgesia. Anesthesiology 98: 156-163, 2003.

20. Luger TJ, Kammerlander C, Benz M, Luger MF and Garoscio I: Peridural anesthesia or ultrasound-guided continuous 3-in-1 block: Which is indicated for analgesia in very elderly patients with hip fracture in the emergency department? Geriatr Orthop Surg Rehabil 3: 121-128, 2012.

21. Foss NB, Kristensen BB, Bundgaard M, Bak M, Heiring C, Virkelyst C, Hougaard S and Kehlet H: Fascia iliaca compartment blockade for acute pain control in hip fracture patients. Anesthesiology 106: 773-778, 2007.

22. Fujihara Y, Fukunishi S, Nishio S, Miura J, Koyanagi S and Yoshiya S: Fascia iliaca compartment block: Its efficacy in pain control for patients with proximal femoral fracture. J Orthop Sci 18: 793-797, 2013.

23. Candal-Couto JJ, McVie JL, Haslam N, Innes AR and Rushmer J: Pre-operative analgesia for patients with femoral neck fractures using a modified fascia iliaca block technique. Injury 36: 505-510, 2005.

24. Dulaney-Cripe E, Hadaway S, Bauman R, Trame C, Smith C, Sillaman B and Laughlin R: A continuous infusion fascia iliaca compartment block in hip fracture patients: A pilot study. J Clin Med Res 4: 45-48, 2012.

25. Viscusi ER and Pappagallo M: A review of opioids for in-hospital pain management. Hosp Pract 40: 149-159, 2012

26. Pedersen SJ, Borgbjerg FM, Schousboe B, Pedersen BD, Jørgensen HL, Duus BR and Lauritzen JB; Hip Fracture Group of Bispebjerg Hospital: A comprehensive hip fracture program reduces complication rates and mortality. J Am Geriatr Soc 56: 1831-1838, 2008

27. Catananti $\mathrm{C}$ and Gambassi G: Pain assessment in the elderly. Surg Oncol 19: 140-148, 2010.

28. Chin RP, Ho CH and Cheung LP: Scheduled analgesic regimen improves rehabilitation after hip fracture surgery. Clin Orthop Relat Res 471: 2349-2360, 2013.

29. Petre BM, Roxbury CR, McCallum JR, Defontes KW III, Belkoff SM and Mears SC: Pain reporting, opiate dosing, and the adverse effects of opiates after hip or knee replacement in patients 60 years old or older. Geriatr Orthop Surg Rehabil 3: 3-7, 2012 .

30. Abou-Setta AM, Beaupre LA, Rashiq S, Dryden DM, Hamm MP, Sadowski CA, Menon MR, Majumdar SR, Wilson DM, Karkhaneh M, et al: Comparative effectiveness of pain management interventions for hip fracture: A systematic review. Ann Intern Med 155: 234-245, 2011

31. Diakomi M, Papaioannou M, Mela A, Kouskouni E and Makris A: Preoperative fascia iliaca compartment block for positioning patients with hip fractures for central nervous blockade: A randomized trial. Reg Anesth Pain Med 39: 394-398, 2014.

32. Birnbaum K, Prescher A, Hessler S and Heller KD: The sensory innervation of the hip joint-an anatomical study. Surg Radio Anat 19: 371-375, 1997.

33. Dalens B, Vanneuville G and Tanguy A: Comparison of the fascia iliaca compartment block with the 3-in-1 block in children. Anesth Analg 69: 705-713, 1989.

34. Szucs S, Iohom G, O'Donnell B, Sajgalik P, Ahmad I, Salah N and Shorten G: Analgesic efficacy of continuous femoral nerve block commenced prior to operative fixation of fractured neck of femur. Perioper Med (Lond) 1: 4, 2012.

35. Yu B, He M, Cai GY, Zou TX and Zhang N: Ultrasound-guided continuous femoral nerve block vs continuous fascia iliaca compartment block for hip replacement in the elderly: A randomized controlled clinical trial (CONSORT). Medicine (Baltimore) 95: e5056, 2016. 
36. Fletcher AK, Rigby AS and Heyes FL: Three-in-one femoral nerve block as analgesia for fractured neck of femur in the emergency department: A randomized, controlled trial. Ann Emerg Med 41: 227-233, 2003.

37. Paul JE, Arya A, Hurlburt L, Cheng J, Thabane L, Tidy A and Murthy Y: Femoral nerve block improves analgesia outcomes after total knee arthroplasty: A meta-analysis of randomized controlled trials. Anesthesiology 113: 1144-1162, 2010.

38. Godoy Monzon D, Iserson KV and Vazquez JA: Single fascia iliaca compartment block for post-hip fracture pain relief. J Emerg Med 32: 257-262, 2007.

39. Inan N, Akin Takmaz S, Iltar S, Yazici I and Basar H: The effects of two different multimodal analgesic regimens in total hip replacement surgery. Agri 21: 69-74, 2009.

40. Capdevila X, Biboulet P, Bouregba M, Barthelet Y, Rubenovitch J and d'Athis F: Comparison of the three-in-one and fascia iliaca compartment blocks in adults: Clinical and radiographic analysis. Anesth Analg 86: 1039-1044, 1998.

41. Newman B, McCarthy L, Thomas PW, May P, Layzell M and Horn K: A comparison of pre-operative nerve stimulator-guided femoral nerve block and fascia iliaca compartment block in patients with a femoral neck fracture. Anaesthesia 68: 899-903, 2013.

42. Deniz S, Atım A, Kürklü M, Çaycı T and Kurt E: Comparison of the postoperative analgesic efficacy of an ultrasound-guided fascia iliaca compartment block versus 3 in 1 block in hip prosthesis surgery. Agri 26: 151-157, 2014.

43. Dolan J, Williams A, Murney E, Smith M and Kenny GN: Ultrasound guided fascia iliaca block: A comparison with the loss of resistance technique. Reg Anesth Pain Med 33: 526-531, 2008 .
44. Ganapathy S, Wasserman RA, Watson JT, Bennett J, Armstrong KP, Stockall CA, Chess DG and MacDonald C: Modified continuous femoral three-in-one block for postoperative pain after total knee arthroplasty. Anesth Analg 89: 1197-1202, 1999

45. Lopez S, Gros T, Bernard N, Plasse C and Capdevila X: Fascia iliaca compartment block for femoral bone fractures in prehospital care. Reg Anesth Pain Med 28: 203-207, 2003.

46. Hebbard P, Ivanusic J and Sha S: Ultrasound-guided supra-inguinal fascia iliaca block: A cadaveric evaluation of a novel approach. Anaesthesia 66: 300-305, 2011.

47. Sanzone AG: Current challenges in pain management in hip fracture patients. J Orthop Trauma 30 (Suppl 1): S1-S5, 2016.

48. Lau TW, Fang C and Leung F: The effectiveness of a geriatric hip fracture clinical pathway in reducing hospital and rehabilitation length of stay and improving short-term mortality rates. Geriatr Orthop Surg Rehabil 4: 3-9, 2013.

49. French DD, Bass E, Bradham DD, Campbell RR and Rubenstein LZ: Rehospitalization after hip fracture: Predictors and prognosis from a national veterans study. J Am Geriatr Soc 56: 705-710, 2008.

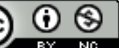

This work is licensed under a Creative Commons Attribution 4.0 International (CC BY-NC 4.0) License 